Objectives: To assess treatment patterns and DMARD discontinuation in RA patients with comorbid lung disease in comparison with other RA patients. Methods: The study included RA Patients enrolled in the Forward Databank with $\geq 1$ year observation after 2000 initiating a DMARD. Forward is a large longitudinal rheumatic disease registry in the US. RA patients' diagnoses were rheumatologist-confirmed, and every 6 months participants completed comprehensive questionnaires regarding symptoms, disease outcomes, medications, and clinical events. Lung disease (LD+) was defined as at least one of the following: emphysema, asthma, bronchitis, COPD, pleural effusion, fibrosis of the lung, "RA lung", or ILD, the later classified by ICD9 codes (England 2019). DMARDs were categorized hierarchically into four groups: csDMARDs, TNFi and NTNFi (bDMARDs), and tsDMARDs. Percentage of patients who initiated different DMARDs were reported for pts with LD+/LD-. Discontinuation was analyzed by Kaplan Meier (KM) curves, log-ranks tests, and Cox regression models using time-varying covariates. Best models were created using backward selection models ( $10 \%$ probability of removal) and pre-defined clinical models.

Results: Of the 21,525 eligible RA patients, $13.8 \%$ had LD+ at the time they initiated a DMARD (follow-up: 69,597 pt-yrs (median $1.9 \mathrm{yrs} / \mathrm{pt}$ )). LD+ patients tended to have more severe RA outcomes and comorbidities. MTX-monotherapy $(48 \%$ vs $44 \%, p<0.001)$ and NTNFi were initiated more frequently in LD+ pts with lower use of TNFi (Figure). DMARD discontinuation rates were higher among LD+ patients for all DMARD groups, but KM curves were only significantly different for csDMARDs and TNFi. Different HRs for LD+ were found depending on the model used ranging from 1.18 to 1.28 , and all models revealed an increased risk of discontinuation for LD+ patients. Compared to csDMARDs, TNFi were more often discontinued (Table). Other variables associated with an increased risk of discontinuation included: $\mathrm{HAQ}$, Rheumatoid Disease (RD) comorbidity index, pain, prior bDMARDs, and csDMARDs.

Conclusion: Different DMARD treatment patterns were found for LD+ patients, who tended to initiate more csDMARD and NTNFi and less likely to initiate a TNFi. LD+ patients were at a higher risk of discontinuation irrespectively of the DMARD treatment, but with greater risk for TNF users.

References:

[1] England BR, et al. Arth Care Res. doi:10.1002/acr.24043.

Figure. DMARD treatment initiators by disease group

Table. Cox models for DMARD discontinuation by stepwise (removal probability $10 \%$ ) and clinical models including DMARD treatment.

\begin{tabular}{lccc}
\hline $\begin{array}{l}\text { Model of DMARD } \\
\text { persistence }\end{array}$ & $\begin{array}{c}\text { Model 1- Stepwise- } \\
\text { Without drugs }\end{array}$ & Model 2 & Model 3 - \\
LD+ vs LD- & 1.18 & 1.28 & Clinical \\
& $(1.08-1.29)$ & $(1.13-1.45)$ & 1.20 \\
TNF vs csDmard & & 1.32 & $1.08-1.34)$ \\
& & $(1.08-1.63)$ & $(1.04-1.44)$ \\
NTNF vs csDmard & 1.13 & 1.13 \\
& & $(0.83-1.52)$ & $(0.90-1.41)$ \\
tsDmard vs csDmard & 1.30 & 1.02 \\
& & $(0.65-2.60)$ & $(0.64-1.62)$
\end{tabular}

*Best models searched/Clinical adjusted for LD+/LD-, DMARDs, age, sex, education, HAQ disability, RD comorbidity index, smoking, pain, glucocorticoids, year of entry, prior bDMARDs and csDMARDs counts and MRC breath scale.

Disclosure of Interests: Sofia Pedro: None declared, Ted Mikuls Grant/ research support from: Horizon Therapeutics, BMS, Consultant of: Pfizer, Joe Zhuo Shareholder of: Bristol-Myers Squibb, Employee of: Bristol-Myers Squibb, Kaleb Michaud: None declared

DOI: 10.1136/annrheumdis-2020-eular.5452

\section{FRI0073 SEROLOGICAL PREDICTORS OF THE SEVERITY OF RHEUMATOID ARTHRITIS RELATED INTERSTITIAL LUNG DISEASE}

S. Rajalingham ${ }^{1}$, S. S. Shaharir ${ }^{1}$, R. Sridharan ${ }^{1} .{ }^{1}$ Universiti Kebangsaan Malaysia Medical Centre, Kuala Lumpur, Malaysia

Background: The most common extra-articular manifestation of rheumatoid arthritis(RA) is interstitial lung disease (ILD). Although pulmonary manifestations in RA encompass the main airway, parenchyma, vasculature and pleura, ILD in particular, is associated with reduced survival. Up to $10 \%$ of RA patients suffer from clinically significant ILD while a substantial proportion have abnormal CT chest findings despite being asymptomatic. There are various biochemical and serological markers to predict the severity of the joints in RA. However, the clinical and laboratory determinants of RA related ILD (RA-ILD) are not well defined owing to the paucity of research data in this regard.
Objectives: The main objective of this study is to determine the correlation between the rheumatoid factor (RF) serotypes and the severity of RA-ILD based on computer tomography (CT) findings.

Methods: We recruited a total of 100 RA patients who were tested for IgA RF, IgG RF and IgM RF and had high resolution CT chest performed. Participants were aged above 18 years, met the 2010 ACR/EULAR RA criteria, had RA for more than 6 months, were non-smokers, not pregnant and had no known chronic lung disease or lung malignancy based on their medical records. Seventy-two patients had ILD changes on HRCT of the chest and were included in this study. The CT images were scored based on a scoring system proposed by Kazerooni et al. Ground glass opacities represented the alveolar findings whereas honeycombing and septal thickening were the interstitial findings. The ground glass and fibrosis scores were on a scale of $0-5$, with higher scores for greater involvement of the lobes. For each subject, the maximum ground glass and fibrosis scores were 25 , respectively.

Results: The frequency of RF positivity was comparable across the 3 serotypes (83.33-84.72\%). The correlation between the clinical variables and the CT scores are listed in Table 1.We found that the the CT scores for ground glass showed significant positive correlation with disease duration $(p=0.047)$ and $\lg A$ RF levels $(p<0.050)$ whereas the fibrosis scores had significant relationship with multiple clinical covariates i.e age $(p=0.004)$, disease duration $(p=0.042)$, IgA RF levels $(p<0.050)$, IgG RF levels $(p=0.041)$ and anti-CCP levels $(p=0.006)$. On multivariate analysis, only IgA RF levels remained significantly $(p<0.05$, standardized beta coefficient $=0.604$ ) associated with the ground glass scores. As for the fibrosis scores, IgA RF levels and age were independent predictors based on multivariate analysis after adjusting for confounders, with $p$ scores of $<0.05$ and 0.02 , respectively.

Table 1. Correlation of CT scores with clinical covariates and antibodies

\begin{tabular}{|c|c|c|c|c|}
\hline \multirow[t]{2}{*}{ Parameters } & \multicolumn{2}{|c|}{ Ground glass } & \multicolumn{2}{|c|}{ Fibrosis } \\
\hline & $r$ & $p$ value & $r$ & $p$ value \\
\hline Age & 0.229 & 0.053 & 0.334 & 0.004 \\
\hline Disease duration & 0.235 & 0.047 & 0.240 & 0.042 \\
\hline Total MSS & 0.058 & 0.628 & 0.224 & 0.145 \\
\hline Cumulative Methotrexate dose & 0.039 & 0.748 & 0.098 & 0.413 \\
\hline $\lg A$ & 0.608 & $<0.05$ & 0.576 & $<0.05$ \\
\hline $\lg M$ & 0.053 & 0.660 & 0.019 & 0.873 \\
\hline $\lg G$ & 0.183 & 0.124 & 0.241 & 0.041 \\
\hline Anti CCP & 0.117 & 0.328 & 0.319 & 0.006 \\
\hline
\end{tabular}

Conclusion: The IgA RF was the only serotype which was independently associated with the severity of RA-ILD.

References:

[1] Bongartz, T., Nannini, C., Medina-Velasquez, Y. F., Achenbach, S. J., Crowson, C. S., Ryu,et al. (2010). Incidence and mortality of interstitial lung disease in rheumatoid arthritis: a population-based study. Arthritis and rheumatism, 62(6), 1583-1591.

[2] Dawson, J. K., Fewins, H. E., Desmond, J., Lynch, M. P., \& Graham, D. R. (2001). Fibrosing alveolitis in patients with rheumatoid arthritis as assessed by high resolution computed tomography, chest radiography, and pulmonary function tests. Thorax, 56(8), 622-627.

[3] Kazerooni, E. A., Martinez, F. J., Flint, A., Jamadar, D. A., Gross, B. H.,et al. (1997). Thin-section CT obtained at 10-mm increments versus limited threelevel thin-section CT for idiopathic pulmonary fibrosis: correlation with pathologic scoring. AJR Am J Roentgenol, 169(4), 977-983.

[4] van der Helm-van Mil, A. H., \& Huizinga, T. W. (2012). The 2010 ACR/ EULAR criteria for rheumatoid arthritis: do they affect the classification or diagnosis of rheumatoid arthritis? Annals of the rheumatic diseases, 71(10), 1596-1598

Disclosure of Interests: Sakthiswary Rajalingham Speakers bureau: Pfizer (500USD), Syahrul Sazliyana Shaharir: None declared, Radhika Sridharan: None declared

DOI: 10.1136/annrheumdis-2020-eular.218

\section{FRI0074 UNDERLYING PROBLEMS AND IMPACT OF DIFFICULT- TO-TREAT RHEUMATOID ARTHRITIS: PRELIMINARY RESULTS OF A CROSS-SECTIONAL CASE-CONTROL STUDY}

N. M. T. Roodenrijs ${ }^{1}$, M. C. Van der Goes ${ }^{1,2}$, P. Welsing ${ }^{1}$, J. Tekstra $^{1}$, F. Lafeber ${ }^{1}$ J. W. G. Jacobs ${ }^{1}$, J. M. Van Laar ${ }^{1} .{ }^{1}$ University Medical Center, Department of Rheumatology \& Clinical Immunology, Utrecht, Netherlands; ${ }^{2}$ Meander Medical Center, Department of Rheumatology, Amersfoort, Netherlands 